\title{
LSST Observations of RR Lyrae Stars for Mapping the Galactic Halo
}

\author{
Hakeem M. Oluseyi ${ }^{1}$, Andrew C. Becker ${ }^{2}$, Christopher C. Culliton ${ }^{1}$, \\ Muhammad Furqan ${ }^{1}$, Keri L. Hoadley ${ }^{1}$, Paul Regencia ${ }^{1}$, \\ Akeem J. Wells ${ }^{1}$, Lynne Jones ${ }^{2}$, Simon Krughoff ${ }^{2}$, Branimir Sesar ${ }^{3}$, \\ and Suzanne Jacoby ${ }^{4}$ \\ ${ }^{1}$ Department of Physics \& Space Sciences, Florida Institute of Technology, \\ 150 W. University Boulevard, Melbourne, Florida 32901 USA \\ email: holuseyi@fit.edu \\ ${ }^{2}$ Astronomy Department, University of Washington \\ 3910 15th Ave NE, Seattle, WA 98195 USA \\ ${ }^{3}$ Astronomy Department, California Institute of Technology \\ 1200 East California Blvd, Pasadena, CA 91125 USA \\ ${ }^{4}$ LSST Corporation, 933 N. Cherry Avenue, Tucson, AZ 85721-0009 USA
}

\begin{abstract}
The Large Synoptic Survey Telescope (LSST) is an anticipated to undertake a 10year, $3 \pi$ steradian survey that promises to observe millions of new periodic variable stars. We report on a study to determine the efficiency of the LSST to recover the light curve properties of RR Lyrae stars. An LSST light curve simulation tool was used to sample input idealized light curves or RR Lyrae stars observed in SDSS Stripe 82 data, returning each as it would have been observed by LSST, including realistic photometric scatter, limiting magnitudes, and telescope downtime. Our results show that the LSST will be capable of mapping the spatial distributions and chemical compositions of halo stellar overdensities using RR Lyrae discovered across $3 \pi$ steradians and out to nearly $1.5 \mathrm{Mpc}$. LSST will thus enable the mapping of halo merger streams, the discovery of new dwarf galaxies, and the mapping galactic halos throughout the Local Group galaxies.
\end{abstract}

Keywords. Keyword1, keyword2, keyword3, etc.jKeywords.txti

\section{Introduction}

Understanding the details of galaxy formation and evolution are major objectives of modern astrophysics. Being both bright and long-lived, RR Lyrae stars provide an abundant means for mapping Galactic halo structure and accretion history. Their utility derives from simple relations correlating observable parameters, such as period, amplitude of pulsation and metallicity, with evolutionary parameters such as luminosity (Marconi et al. 2006). Detections of overdensities of RR Lyrae stars have been successfully used to identify substructure within our own Galactic halo (Ivezić 2000; Ivezić et al. 2005; Sesar et al. 2007; Sesar et al. 2010; Keller et al. 2008; Watkins et al. 2009). Metallicity estimates of detected RR Lyrae stars additionally constrain Galaxy accretion models that predict a difference in chemical composition between the inner (old accretion) and outer (recent accretion) halo (Bullock \& Johnston 2005; Johnson et al. 2008; Szczygie et al. 2009). However, current RR Lyrae observations have been over a limited area, providing only volumetric slices through any extant Galactic halo substructure. The complex and overlapping morphologies of accreted substructures seen in galaxy accretion simulations (Cooper et al. 2010; Johnson et al. 2008) require deep full-sky surveys like the LSST to trace and disentangle the observed halo streams. 


\section{The Large Synoptic Survey Telescope}

The Large Synoptic Survey Telescope is an anticipated 8.4-meter telescope with a 9.6$\mathrm{deg}^{2}$ field of view that will repeatedly image the entire available sky every 3 to 4 days. The filter set includes 6 ugrizY passbands, the first 5 of which are very similar to the SDSS ugriz bands (Fukugita et al. (996). The core LSST observing strategy is to take two backto-back 15-second exposures in a given filter and to return to the same pointing within 15-60 minutes ([Ivezic et al. 2008). This Universal Cadence (UC), which will consume the majority $(\sim 90 \%)$ of LSST's observing time, is designed to image $\sim 20,000$ square degrees of the sky with a total of $\sim 1000$ observations distributed amongst all filters. Deep Drilling (DD) observations allocate 10 minutes of continuous 15-second exposure per night, distributed amongst filters on a 5-day cycle.

To explore LSST's ability to recover light curve shape information as a function of distance, we realize each idealized light curve used in the simulations over a range of mean $g$-band magnitudes, from 20.0 to 27.0 in steps of 0.5 magnitudes. Finally, we explore the evolution of LSST's state of knowledge of each star by exploring subsets of the ten-year simulated light curves in one year increments. In total this yields $40 \mathrm{RR}$ Lyrae stars' light curves $\times 6$ filters $\times 1007$ field centers $\times 15 g$-band magnitude bins $\times$ 10 sub-surveys, for just over 36 million light curves that have gone into this study.

\section{Period Recovery}

Amongst the first steps in classifying variability is searching for periodicity in the observed light curve. To this end we have run period-finding software on each of our 36 million simulated light curves. We used the variable span Supersmoother algorithm of ? for period estimation. To ascertain whether or not Supersmoother recovered the known input period, we require that the product of the fractional misfit in the recovered period and the number of pulsation cycles be less than some fraction of a cycle:

$$
N \times \sigma_{P} \leqslant \delta \phi_{\max }
$$

where $\sigma_{P} \equiv\left|P_{S S}-P_{i n}\right| / P_{i n}, P_{i n}$ is the known input period, and $P_{S S}$ is the fitted Supersmoother period. The number of cycles $N$ is given by the ratio of the survey length $\Delta t$ and the period of variability $P_{i n}$, and $\delta \phi_{\max }$ is the maximum allowed phase offset after period-folding $N$ cycles. Our criterion for period recovery is thus given by,

$$
\frac{\left|P_{S S}-P_{i n}\right|}{P_{i n}} \leqslant \frac{\delta \phi_{\max } P_{i n}}{\Delta t} .
$$

To estimate a reasonable value for $\delta \phi_{\max }$, we folded several 10-year light curves with varying mis-fits $\delta P$ on the period (where $\delta P \equiv \sigma_{P} / P_{i n}=\delta \phi_{\max } / \Delta t$ ). Values of $\delta \phi_{\max } / \Delta t<$ $10^{-5}$ days $^{-1}$ (or $\delta \phi_{\max }=0.037,1 / 27^{t h}$ of a cycle) were found to yield light curves with well-resolved minima in the RRab stars. Thus, in terms of the input period $P_{i n}$ and the recovered period $P_{S S}$, our criterion for successful period recovery may be written as:

$$
\frac{\left|P_{S S}-P_{i n}\right|}{P_{i n}^{2}} \leqslant 10^{-5} \text { days }^{-1} .
$$

The criterion defined in Equation 3.3 for a 10-year survey, was applied to periods recovered from the $g, r$, and $i$ passbands. A successful period recovery is defined by having at least 2 out of these 3 periods within the tolerance specified in Equation 3.3. 


\section{Results and Discussion}

\subsection{Opsim 1_29 Universal Cadence}

Covering 20,000 $\mathrm{deg}^{2}$ and consuming $90 \%$ of LSST's observing time, the UC constitutes the bulk of LSST data. Shown in Figure 1 is the surfaces and plot of period recovery for the UC fields. The surface is for the combined set of RRab and RRc stars and includes all six passbands of data. The 2-dimensional plot provides the 1, 2, 5, and 10 year recovery curves and shows the results for all stars as solid lines and the results for RRab stars as dashed lines. The larger amplitudes of RRab stars allow substantially higher period recovery at fainter magnitudes $(\langle g\rangle \geqslant 21.0)$ after 5 years.

These results show that after five years, UC fields will have nearly $95 \%$ recovery out to $\sim 200 \mathrm{kpc}(\langle g\rangle=21.5)$. Doubling the length of the survey pushes a given completeness level approximately 1.5 magnitudes deeper in UC fields. For stars fainter than $\langle g\rangle=22.5$ the recovery efficiency for the 10-year light curves is $\sim 30 \%-40 \%$ better than for the five-year light curves, decreasing to zero at $\langle g\rangle \approx 26.0$.
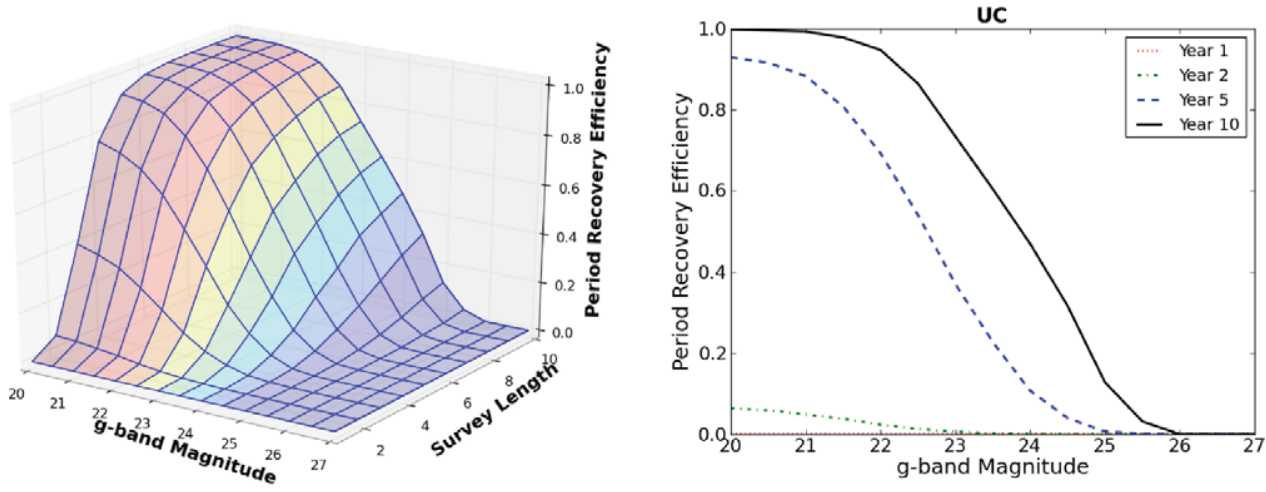

Figure 1. : Left: Surface of period recovery efficiency for the UC fields. Right: Recovery curves of UC after 1, 2, 5, and 10 years of LSST observations. The solid lines indicate the results for all stars and the dashed line indicates the results for RRab stars.

\subsection{Opsim 1_29 Deep Drilling Cadence}

The DD fields, which constitute $70 \mathrm{deg}^{2}$, are visited most frequently among LSST's five cadences, with 40 total observations per night. Figure 2 shows the frequency with which the period recovery criterion was met as a function of LSST survey length and RR Lyrae $g$-band magnitude. In the left panel we show the entire recovery surface for all magnitudes and survey lengths. In the right panel, we show slices through this surface for years $1,2,5,10$ of the survey. The solid line indicates the recovery rate for the combined set of RRab and RRc stars, while the dashed line indicates the recovery rate for RRab stars only. By the second year, $\sim 85 \%$ of RR Lyrae stars within $\sim 160 \mathrm{kpc}$ $(\langle g\rangle=21.0)$ will have their periods recovered to within $10^{-5}$ by LSST photometry, with successful recoveries extending to $\geqslant 790 \mathrm{kpc}$. By year five, there is near $100 \%$ recovery to $\sim 250 \mathrm{kpc}(\langle g\rangle=22.0)$, and by year 10 , to $\sim 400 \mathrm{kpc}(\langle g\rangle=23.0)$. For RRab, these limits extend 0.5 magnitude fainter. A substantial fraction of stars beyond $1.2 \mathrm{Mpc}$ will have their periods successfully recovered after 10 years, yielding the opportunity to detect substructure through RR Lyrae overdensities throughout the Local Group. Recovery falls precipitously by $\langle g\rangle \approx 26.0(1.6 \mathrm{Mpc})$. 

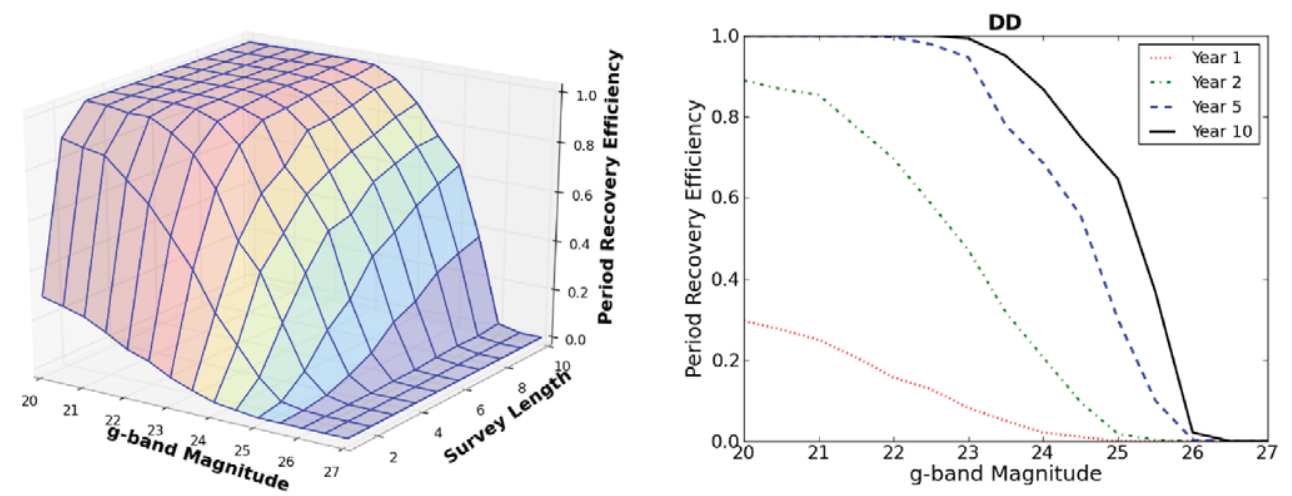

Figure 2. : Left: Surface of period recovery efficiency for the DD fields. Right: Recovery curves of DD after 1, 2, 5, and 10 years of LSST observations. The solid lines indicate the results for all stars and the dashed line indicates the results for RRab stars.

\section{Conclusions}

Our investigation of period recovery shows that the majority of Galactic halo RR Lyrae periods will be recovered in 2 years of Deep Drilling (DD) observations, and in 5 years of Universal Cadence (UC) observations. To recover the periods of RR Lyrae at $\sim \mathrm{Mpc}$ distances requires a minimum of 5 years of DD observations. A slight modification of the DD cadence, incorporating a field revisit within a night, may decrease the amount of time it takes to recover the periods of such short period objects. If the saturation of science occurs more rapidly, the DD field centers may be moved after a couple of years to accomplish similar science at a different pointing.

The results shown here indicate that the LSST will possess unprecedented capabilities for measuring the Milky Way's history of Galactic mergers by mapping the spatial distributions and chemical compositions of halo stellar overdensities, using RR Lyrae discovered across $3 \pi$ steradians, and out to $1 \mathrm{Mpc}$ and beyond. This will enable the mapping of halo merger streams, placing strong constraints on galaxy formation models; the discovery of new dwarf galaxies in the Galactic halo and Local Group, thus addressing the "missing satellites" problem; and the mapping of the halos of Local Group galaxies, helping to constrain the general processes involved in galaxy formation and evolution in $\Lambda \mathrm{CDM}$ cosmology.

\section{References}

Bullock, J. S. \& Johnston, K. V. 2005, ApJ, 635, 931

Cooper, A. P., Cole, S., Frenk, C. S., White, S. D. M., Helly, J., Benson, A. J., De Lucia, G.,Helmi, A., Jenkins, A., Navarro, J. F., Springel, V., \& Wang, J. 2010, MNRAS, 406, 744

Fukugita, M., Ichikawa, T., Gunn, J. E., Doi, M., Shimasaku, K., \& Schneider, D. P. 1996, AJ, 111,1748

Ivezic, Z., Tyson, J. A., Allsman, R., Andrew, J., \& Angel, R., for the LSST Collaboration. 2008, ArXiv e-prints 0805.23661

Ivezić, Z., Vivas, A. K., Lupton, R. H., \& Zinn, R. 2005, AJ, 129, 1096

Ivezić, Z. et al. 2000, AJ, 120, 963

Johnston, K. V., Bullock, J. S., Sharma, S., Font, A., Robertson, B. E., \& Leitner, S. N. 2008, ApJ, 689, 936

Keller, S. C., Murphy, S., Prior, S., Da Costa, G., \& Schmidt, B. 2008 ApJ, 678, 851 
Marconi, M., Cignoni, M., Di Criscienzo, M., Ripepi, V., Castelli, F., Musella, I., \& Ruoppo, A. 2006, MNRAS, 371, 1503

Sesar, B. et al. 2010, ApJ, 708, 717

Sesar, B. et al. 2007, AJ, 134, 2236

Szczygie 1. D. M., Pojmański, G., \& Pilecki, B. 2009, Acta Astronomica, 59, 137

Watkins, L. L. et al. 2009, MNRAS, 398, 1757 